\title{
Best Possible Bounds on the Weighted \\ Path Length of Optimum Binary Search \\ Trees
}

by

Kurt Mehlhorn

\section{Abstract :}

We derive upper and lower bounds for the weighted path length $\mathrm{P}$ opt of optimum binary search trees. In particular,

$$
1 / \log 3 \mathrm{H} \leq \mathrm{P}_{\text {opt }} \leq 2+\mathrm{H}
$$

where $H$ is the entropy of the frequency distribution. We also present an approximation algorithm which constructs nearly optimal trees.

\section{Introduction}

"One of the popular methods for retrieving information by its 'name' is to store the names in a binary tree. We are given $n$ names $B_{1}, B_{2}, \ldots, B_{n}$ and $2 n+1$ frequencies $\beta_{1}, \ldots, \beta_{n}, \alpha_{0}, \ldots, \alpha_{n}$ with $\Sigma \beta_{i}+\Sigma \alpha_{j}=1$. Here $\beta_{i}$ is the frequency of encountering name $B_{i}$, and $\alpha_{j}$ is the frequency of encountering a name which lies between $B_{j}$ and $B_{j+1}$, (a name in the interval $\left.\left(B_{j}, B_{j+1}\right)\right) \alpha_{0}$ and $\alpha_{n}$ have obvious interpretations". ([5]).

Fachbereich 10

Angewandte Mathematik und Informatik

Universität des Saarlandes, D 66 Saarbrücken 
A binary search tree $\mathrm{I}$ is a tree with $\mathrm{n}$ interior nodes ( nodes having two sors, , wich we denote by circles, and $\mathrm{n}+1$ leaves, which ve denote by squares. The interior nodes are labelled by the $B_{i}$ in increasing order from left to right and the leaves are labelled by the intorvals $\left(B_{j}, B_{j+1}\right)$ in increasing order from left to right. Let $b_{i}$ be the distance of interior node $B_{j}$. from the root and let $a_{j}$ be the distance of leaf $\left(B_{j}, B_{j+1}\right)$ from the root. To retrieve a name $x, b_{i}+1$ comparisons are needed if $x=B_{i}$ and $a_{j}$ comparisons are required if $B_{j}<x<B_{j+1}$. Therefore we define the weighted poth lenght of tree $\mathrm{T}$ as :

$$
P=\sum_{i=1}^{n} B_{i}\left(b_{i}+1\right)+\sum_{j=0}^{n} a_{j} a_{j}
$$

It is equal to the expected number of comparisons needed to retrieve a name.

The following two problems are among the most important in this area $([5])$.

a) Prove good lower and upper bounds for the weighted path length of optimum binary search trces, i.e. the trees with minimal weighted path length. Such bounds would provide us with a simple a-priori test for the performance of binary search trees.

b) Design efficient algorithms for constructing optimal ( or nearly so, binary search trees.

In this paper, we attempt to solve both problems. 


\section{$\tau I$. Upper Bounds}

In this section, we will show that $1+\sum \alpha_{j}+\mathrm{H} \quad-\mathrm{H}=$ $-\sum B_{i} \log \beta_{i}-\sum \alpha_{j} \log \alpha_{j}$ is the entropy of the frequency distribution -- is an upper bound on the veighted path length Popt of the optimum binary search tree. Furthermore this bound is best possible among the bounds of the form $c_{1} \sum \beta_{i}+c_{2} \sum \alpha_{j}+c_{3} \cdot H$.

We prove the upper bound by acscribing and analyzing an approximation algorithm. This algorithm constructs binary search trees in a top-down fashion. It uses bisection on the set

$$
\begin{aligned}
& \left\{s_{i} ; s_{i}=\sum_{p=0}^{i-1}\left(\alpha_{p}+\beta_{p}\right)+\beta_{i}+\alpha_{i} / 2\right. \\
& \text { and } 0 \leq i \leq n\}, i . e .
\end{aligned}
$$

the root $k$ is determined such that $s_{k-1} \leqslant 1 / 2$ and $s_{k} \geq 1 / 2$. It proceeds then recursively on the subsets $\left\{s_{i} ; i \leq k-1\right\}$ and $\left\{s_{i} ; i 2 k\right\}$.

The main program

begin

$$
\begin{aligned}
& \text { Let } s_{i}+\sum_{p=0}^{i-1}\left(\alpha_{p}+\beta_{p}\right)+\beta_{i}+\alpha_{i} / 2 \text { for } 0 \leq i \leq n \\
& \text { construct-tree }(0, n, 0,1)
\end{aligned}
$$

end

uses the recursive procedure construct-tree.

construct-tree $(i, j, c u t, \ell)$;

conment we assume that the actual parameiers of any call of construct-tree satisfy the following conditions.

(1) $i$ and $j$ are intogers with $0 \leq i<j \leq n$, 
(2) 2 is an integer with $\& \geq 1$,

(3) cut $=\sum_{p=1}^{2-1} x_{p} 2^{-p}$ with $x_{p} \varepsilon\{0,1\}$ for al1 $p$,

(4) cut $\leqslant s_{i} \leq s_{j} \leq$ cut $+2^{-\ell+1}$.

A call construct-tree $(i, j,-,-$,$) will construct a binary.$ search tree for the nodes $(i+1, \ldots,(j)$ and the leaves is $\ldots, i j$

beoin

if $i+1=j(\operatorname{case} A)$

then return the tree

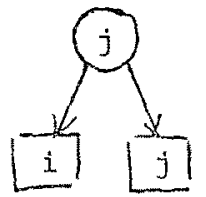

else commont we determine the root so as to bisect the interval

(cut, cut $+2^{-t+1}$ )

\section{begin}

determine $k$ such that

(5) $i<k \leq j$

(6) $k=i+1$ or $s_{k-1} \leq$ cut $+2^{-\ell}$

(7) $k=j$ or $s_{k} \geq$ cut $+2^{-\ell}$

comment $k$ exists because the actual parameters are supposed to satisfy condition (4);

if $k=1+1$ ( case $B)$

then return the tree

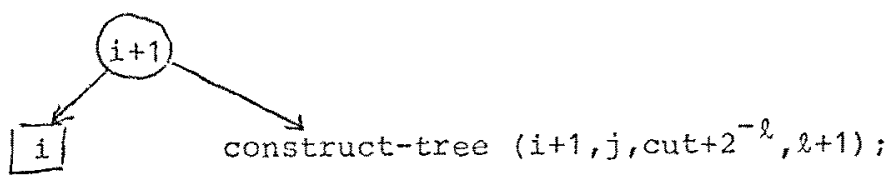

$\underline{i E} \mathrm{k}=\mathrm{j}(\operatorname{cose} \mathrm{C})$

then return the tree

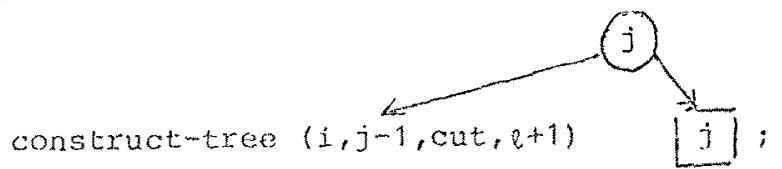


if $i+1<k<j($ case $D)$

then return the tree

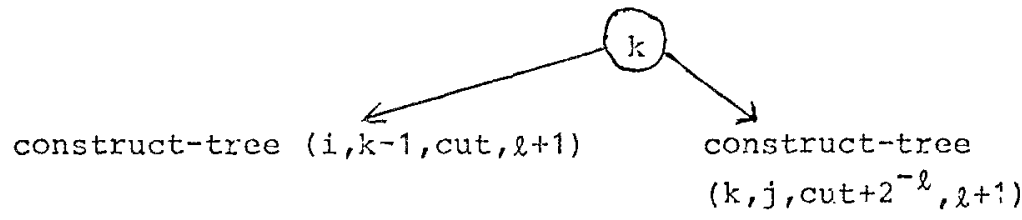

end

end.

Lemma :

The approximation algorithm constructs a binary search tree whose weighted path length Papprox is bounded above by

$$
1+\sum \alpha_{j}+H
$$

The algorithm can be implemented to work in $0(n \log n)$ units of time and $O(n)$ units of space.

\section{proof:}

We state several simple facts.

\section{Fact 1:}

If the actual parameters of a call construct-tree ( $i, j$, cut, $f$ ) satisfy conditions (1) to (4) and $i+i \frac{1}{T} j$ then a $k$ satisfying conditions (5) to (7) exists and the actual parameters of the recursive calls of construct-tree iniated by this call again satisfy conditions (1) to (4).

\section{Fact $2:$}

The actual parameters of evory call of construct-tree satisfy conditions (1) to (4) ( if the arouments of the top-level call (3) 1 . 
We say that node (h) (leaf [h] respectively) is constructed.

by call construct-tree $(1, j$, cut, $l)$ if $h=j(h=i$ or $h=j)$ and case $A$ is taken or if $h=i+1(h=i)$ and case $B$ is taken or if $h=j(h=j)$ and case $c$ is taken or if $h=k$ and case $D$ is taken. Let $b_{i}$ be the depth of node (i) and let $a_{j}$ be the depth of leaf $\bar{j}$ in the tree returned by the call construct-tree $(0, n, o, 1)$

\section{Eact $3:$}

If node $h$ ( leaf $h$ ) is constructed by the call constructtree $(i, j$, cut, $\ell)$ then $b_{h}+1=\ell\left(a_{h}=\ell\right)$.

\section{Fact $4:$}

If node (h) ( leaf h ) is constructed by the call constructtree $(i, j$, cut, $l)$ then $B_{h} \leqslant 2^{-\ell+1}\left(\alpha_{h} \leq 2^{-\ell+2}\right)$.

\section{Pact 5:}

The weighted path length $P_{\text {approx }}$ of the tree constructed by the approximation algorithm is bounded above by $1+\sum \alpha_{j}+H$.

We sketch now an efficient implementation of our approximation algorithm. The complexity of the algorithm is determined by the complexity of the search for $k$. If we search for $k$ simultaneously from both ends, i.e. try $k=1+1, k=j, k=i+2, k=j-1$, ... successively, then the complexity of this search is 0 ( min $(k-i, j-k+1))$. Hence ve get the following recurrence relation for the complexity of construct-tree ( as a function of j -1. ). 
$T(m) \leq \begin{cases}0 & \text { if } n=0 \text { (by definition) } \\ c_{1} & \text { if } n=1 \\ \max _{0<k<m / 2}[\mathrm{~T}(k)+\mathrm{T} & \left.(m-k+1)+c_{2}(k+i)\right] \text { otherwise }\end{cases}$

for some constants $c_{1}, c_{2}$.

\section{Eact 7 :}

The recurrence relation ( $*$ ) has a solution $T(n) \leq 0(n \log n)$.

\section{Fact $8:$}

The approximation algorithm can be implemented to work in $O(n \log n)$ units of time and $O(n)$ units of space.

$$
\text { q. e. d. }
$$

\section{Theorem 1 :}

Let $\alpha_{0}, \beta_{1}, \alpha_{1}, \ldots, \beta_{n}, \alpha_{n}$ be any frequency distribution, let $P_{\text {opt }}$ be the weighted path length of the optimum binary search tree for this distribution, let $P_{\text {approx }}$ be the weighted path length of the tree constructed by the approximation algorithm, and let $I z=-\sum \beta_{i} \log \beta_{i}-\sum \alpha_{j} \log \alpha_{j}$ be the entropy of the frequency distribution. Then

$$
\mathrm{P}_{\text {opt }} \leq \mathrm{P}_{\text {approx }} \leq 1+\sum \alpha_{j}+\mathrm{H} \text {. }
$$

Furthermore, this upper bounds is best possible in the following sense : If $c_{1} \sum \beta_{j}+c_{2} \sum a_{j}+c_{3} \cdot H$ is an upper bound on Fopt then $c_{1} \geq 1, c_{2} \geq 2$, and $c_{3} \geq 1$.

\section{proof:}

The first part of the theorem follows from the preceding lemma. The second part is proven by oxhibiting suitable frequency distributions. 
$c_{1} \geq 1$ : Take $n=1, \alpha_{0}=\alpha_{1}=0$ and $\beta_{1}=1$.

$c_{2} \geq 2$ : Take $n=2, \alpha_{0}=\alpha_{2}=\beta_{1}=\beta_{2}=0, \alpha_{1}=1$.

$c_{3} \geq 1$ : Take $n=2^{k}-1, \beta_{1}=0$ for all $i$ and $\alpha_{j}=2^{-k}$ for all $j$.

It is easy to see that the complete binary tree is the

optimal binary search tree for this distribution. Thus

$\mathrm{H}=\log \mathrm{n}+1=\mathrm{k}=\sum_{\text {leaves }} 1 / 2^{\mathrm{k}} \cdot \mathrm{k}=\mathrm{P}_{\mathrm{opt}}$

a. e. a.

E.N. Gilbert and E.F. Moore (1) proved this theorem in the special case that all internal nodes have weight zero

( $i . e . B_{i}=$ o for all $i$ ). Theix proof suggest the approximation algorithm which we presented above. Other " rules of thumb " are aiscussed in $[7,8]$; we prove in $[7]$ that the strategy "choose the root so as to equalize the total weights of the left and right subtree as much as poosible "yields trees whose weighted path length is bounded above by $2+1,44 \cdot \mathrm{H}$.

c.p. Schnorr improves this bound to $3+1,07 \cdot \mathrm{H}$ in $[8]$. In the case that all internal nodes have weight 0 an algorithm due to T.C. Hu and A.C. Tucker [3] finds the optimum binary search tree in $O(n \log n)$ units of time and $O(n)$ units of space. In the general case, D.E. Knuth shows how to find the optimum tree in $0\left(n^{2}\right)$ units of time and $0\left(n^{2}\right)$ units of space $[5]$.

\section{Lower Bounds:}

We turn now to lower lounds. Again we will exhibit bounds which are best possible. upper and lower bounds differ only by a constant factor; thus they define a naxrow interval containing the weichted path longth of the optimum (and the nearly optimal) search trec. This perrits a simple a-priori test for the perfor- 
mance of binary search trees.

\section{Theorem 2:}

a) $([1]$ ) : If all interpal nodes have weight zero,

$$
\begin{aligned}
& \left(\text { all } B_{i}=0 ;\right. \text { then } \\
& H \leq P_{\text {opt }}
\end{aligned}
$$

b) Otherwise

$$
1 / \log 3 \text { H } \leq P_{\text {opt }}
$$

c) Both bounds are best possible in the following sense : If $c_{1} \sum \beta_{i}+c_{2} \sum \alpha_{j}+c_{3} H$ is a lower bound on the weighted path length of optimum binary search trees then $c_{3} \leq 1$ in case a) and $c_{3} \leq 1 / \log 3$ otherwise. Furthermore, if $c_{3}=1$ in case a) or $c_{3}=1 / \log 3$ in case b) then $c_{1}, c_{2} \leq 0$.

d) Both bounds are sharp for infinitely many distributions.

\section{Proof:}

Let $B_{0}, \alpha_{1}, \ldots, B_{n}, \alpha_{n}$ be any frequency distribution and let $\mathrm{T}_{\text {opt }}$ be the optinum binary search tree for this distribution, let $b_{i}\left(a_{j}\right)$ be the distance of node (i) (leaf $[j]$ ) from the root, and let $p_{\text {opt }}=\sum B_{i}\left(b_{i}+1\right)+\sum \alpha_{j} a_{j}$ be the weighted path length of $\mathrm{T}_{\text {opt }}$.

We define new Erequency distributions. If all internal node have weight o ( all $B_{i}=0$ ) then define

$$
\begin{aligned}
& \beta_{i}^{\prime}=0 \text { for } 1 \leq i \leq n \\
& a_{j}^{\prime}=2^{-a_{j}} \text { for } 0 \leq j \leq n,
\end{aligned}
$$

otherwise define

$$
\begin{aligned}
& B_{i}^{\prime \prime}=3^{-\left(b_{i}+1\right)} \text { for } 1 \leq i \leq n \\
& \alpha_{j}^{\prime \prime}=3^{-a_{j}} \text { for } 0 \leq j \leq n .
\end{aligned}
$$

It is easy to see that $\sum \beta_{i}^{\prime}+\sum \alpha_{j}^{\prime}=\sum \beta_{i}^{\prime \prime}+\sum \alpha_{j}^{\prime \prime}=1$. 
The following inequality is well-known ( cf. [4]). If $p_{1}, \ldots, p_{n}$ and $q_{1}, \ldots, q_{n}$ are two frequency distributions $\left(\sum p_{i}=\sum q_{i}=1\right)$ then

$-\sum p_{i} \log p_{i} \leq-\sum p_{i} \log q_{i}$

with equality if and only if $p_{i}=q_{i}$ for all-i.

It yields in our case :

$$
\begin{aligned}
H & =\sum \alpha_{j} \log 1 / \alpha_{j} \\
& \leq \sum \alpha_{j} \log 1 / \alpha_{j} \\
& \leq \quad \sum \alpha_{j} \log 2^{a_{j}}=P_{\text {opt }}
\end{aligned}
$$

if all internal nodes have weight zero and

$$
\begin{aligned}
H & =\sum \beta_{i} \log 1 / \beta_{i}+\sum \alpha_{j} \log 1 / \alpha_{j} \\
& \leq \sum \beta_{i} \log 1 / \beta_{i}^{\prime \prime}+\sum \alpha_{j} \log 1 / \alpha_{j}^{\prime \prime} \\
& \leq \sum \beta_{i} \log 3^{\left(b_{i}+1\right)}+\sum \alpha_{j} \log 3^{a_{j}} \\
& \leq(\log 3) P_{\text {opt }}
\end{aligned}
$$

otherwise with equality if $\beta_{i}=\beta_{i}^{\prime}\left(\beta_{i}^{\prime \prime}\right)$ and $\alpha_{j}=\alpha_{j}^{\prime}\left(\alpha_{j}^{\prime}\right)$ for all $i$ and $j$.

Assume now that $\beta_{i}=\beta_{i}^{\prime}\left(\beta_{i}^{\prime}\right)$ and $\alpha_{j}=\alpha_{j}^{\prime}\left(\alpha_{j}^{\prime \prime}\right)$ for all $i$ and $j$. Then it is easy to see that the approximation algorithm of section II constructs $\mathrm{T}_{\text {opt }}$. Thus $\mathrm{P}_{\text {opt }}=\mathrm{II}$ if all internal nodes have weight 0 and $\mathrm{P}_{\text {opt }}=1 / \log 3 \mathrm{H}$ otherwise. The lower bounds stated above are hence sharp for infinitely many distributions.

Part c) of the theorem is now inferred easily. The details are left to the reader. 
IV. Conclusion

We proved that $P_{\text {opt' }}$ the weighted path length of the optimum binary search tree, lies in the following interval

$1 / \log 3 \mathrm{H} \leq \mathrm{P}_{\text {opc }} \leq 1+\mathrm{H}$ if all leaves have weight $\mathrm{O}$

$\mathrm{H} \leq \mathrm{P}_{\text {opt }} \leq 2+\mathrm{H}$ if all internal nodus have weight $\mathrm{O}$

$1 / \log 3 \mathrm{H} \leq \mathrm{P}_{\text {opt }} \leq 1+\sum \alpha_{\mathrm{j}}+\mathrm{H}$ otherwise.

All bounds are best possible. Furthermore, we exhibited an approximation algorithm which constructs trees, whose path legth lies in the intervals stated above, and which can be implemented to work in $O$ ( in log) units of time and $O$ ( $n$ ) units of space.

\section{Acknowledciement :}

I want to thank Prof. C.P, Schnorr for many extremely stimulating discussions on the subject of this paper.

\section{Bibliography:}

1. E.N. Gilbert and E.F. Moore: Bell system Tecinn. Journal 38

2. T.C. Hu and K.C. Tan : Least upper Bound on the Cost of optimum Binary Search Trees, Acta Informatica, 1, 307-310 (1972).

3. T.C. Hu and A.C. Tucker : Optimal Computer search Trees and variable length alphabetic codes, siam J. Applied Math. 21, $514-532,(1971)$.

4. $\frac{\text { T. }}{\mathrm{B} I}$ Kameda and $\mathrm{K}$. Weihrauch: Einführung in die Kodierungstheorie,

5. D.E. Knuth: Optimum Binary Search Trees, Acta Informatica, 1,

6. D.E. Knuth: The Art of Computer Programing, Vol. 3.

7. K. Mehlhorn : Nearly Optimum Binary Search Trees, preorint, Fachborejeir To, Universitit dos Samelanics, 1974 .

8. C.P. Schnorr : I'o Algorithms for Nearly optinal pinaiy search ixecs, Preprint, Fachbereich Mathematik, Jntversitit Prarkfurt, 1974. 